\title{
Mathematical and numerical approaches for multiscale problems
}

This volume collects lecture notes and review articles on various topics addressed during:

- the CEA-EDF-Inria winter school on the theoretical and numerical aspects of periodic and stochastic homogenization (Rocquencourt, Dec. 13-16, 2010)

- the GDR-Chant summer school on the modeling and analysis of problems with rough surfaces and defects (Vienna, Aug. 23-27, 2010).

The first contribution is an introduction to deterministic homogenization techniques by Grégoire Allaire. The theoretical bases (two-scale methods for the periodic setting, G- and H-convergence) are detailed, as well as applications to Stokes flows and diffusion equations. Numerical methods also are briefly presented.

The state-of-the-art numerical homogenization methods for linear elliptic equations are then reviewed and analyzed in the lecture notes by Antoine Gloria.

The third contribution is a review article by Dorin Bucur, Anne-Laure Dalibard and David Gérard-Varet on wall laws for viscous flows near rough surfaces of small amplitude and wavelength.

Questions concerning the construction of boundary layer correctors for the conductivity problem for periodic rough thin layers, and the explicit characterization of the generalized polarization tensor, are addressed by Clair Poignard in the fourth contribution to this volume.

Lastly, a numerical study of acoustic multiperforated plates is provided by Abderrahmane Bendali, M'Barek Fares, Sophie Laurens and Sébastien Tordeux.

Eric Cancès and Stphane Labbé 